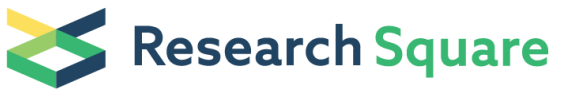 \\ Preprints are preliminary reports that have not undergone peer review. \\ They should not be considered conclusive, used to inform clinical practice, or referenced by the media as validated information.
}

\section{Effectiveness of Deep Hyperthermia Combined with Dynamic Traction in the Treatment of Knee Osteoarthritis}

\section{Lichun Wang ( $\sim$ Wanglichun1976@163.com )}

Department of Neurorehabilitation, Rehabilitation Hospital Area of Cangzhou Integrated Traditional Chinese and Western Medicine Hospital

\section{Liu LV}

Rehabilitation Department, Rehabilitation Hospital Area of Cangzhou Integrated Traditional Chinese and Western Medicine Hospital

\section{Hongli Zhang}

Department of Neurorehabilitation, Rehabilitation Hospital Area of Cangzhou Integrated Traditional Chinese and Western Medicine Hospital

\section{Hua Chen}

Seven Orthopedic Departments, Cangzhou Integrated Traditional Chinese and Western Medicine Hospital

\section{Fei Li}

Department of Neurorehabilitation, Rehabilitation Hospital Area of Cangzhou Integrated Traditional Chinese and Western Medicine Hospital

\section{Jianyong Zhao}

Dean, Cangzhou Integrated traditional Chinese and Western Medicine Hospital

\section{Kaixuan Yao}

Rehabilitation Department, Rehabilitation Hospital Area of Cangzhou Integrated Traditional Chinese and Western Medicine Hospital

\section{Xiaochen Feng}

Rehabilitation Department, Rehabilitation Hospital Area of Cangzhou Integrated Traditional Chinese and Western Medicine Hospital

\section{Research Article}

Keywords: knee joint, osteoarthritis, knee, rehabilitation, high frequency ultrasound, hyperthermia, induced, traction

Posted Date: February 1st, 2021

DOI: https://doi.org/10.21203/rs.3.rs-151216/v1 
License: (a) (i) This work is licensed under a Creative Commons Attribution 4.0 International License. Read Full License 


\section{Abstract}

Background: Different treatments with varying clinical efficacies have been applied to treat knee osteoarthritis (KOA). This study observes the effectiveness of deep hyperthermia combined with dynamic traction in KOA treatment.

Methods: Deep hyperthermia and dynamic traction was administered to 60 patients with KOA. The pretreatment and 20- and 40-day post-treatment visual analog scale (VAS) and Lysholm score, knee joint cavity effusion, synovium thickness, and synovial blood flow signal (by high-frequency ultrasound) of the patients were recorded. The pretreatment and 20 - and 40-day post-treatment Lysholm scores were $47.17 \pm$ $5.07,63.22 \pm 3.44$, and $81.98 \pm 3.30(P<0.001)$, respectively.

Results: The pretreatment and 20- and 40-day post-treatment VAS scores were $5.67 \pm 0.91,2.92 \pm 0.81$, and $0.60 \pm 0.72(P<0.001)$, respectively. The pretreatment and 20 - and 40 -day post-treatment articular effusion was $5.41 \pm 0.46 \mathrm{~mm}, 4.34 \pm 0.34 \mathrm{~mm}$, and $2.32 \pm 0.32 \mathrm{~mm}(P<0.001)$, respectively. The pretreatment and 20- and 40-day post-treatment synovial thickness was $3.44 \pm 0.31 \mathrm{~mm}, 2.79 \pm 0.15 \mathrm{~mm}$, and $2.32 \pm 0.32$ $\mathrm{mm}(\mathrm{P}<0.001)$, respectively. The pretreatment and 20 - and 40 -day post-treatment grades of synovial blood flow signal exhibited a significant difference $(P<0.001)$.

Conclusion: Deep hyperthermia combined with dynamic traction can significantly improve the clinical symptoms and pain degree in KOA, reduce joint cavity effusion, synovium thickness, and internal blood flow, and the effect improves with time. The changes in soft tissues observed by high-frequency ultrasound are reliable and consistent with clinical signs.

\section{Background}

The knee joint is one of the largest synovial joints in the body and is composed of osseus structures, cartilage, ligaments, and synovial membrane. Due to the high amount of stress and wear to the knee joint it is highly likely to be affected by painful conditions such as osteoarthritis (OA). OA is a common joint disorder and the most common cause of hip and knee replacement. It is one of the leading causes of disability and has a significant impact on physical and mental well-being [1]. The prevalence of symptomatic knee OA (KOA) in adults above 60 years of age is approximately $10 \%$ in men and $13 \%$ in women [2]. Various risk factors for KOA have been identified; these include female gender, AfricanAmerican race, genetic predisposition, obesity, dietary exposure, physical activity, and high bone density and mass, and occupational factors such as jobs requiring heavy manual labor. The pathogeneses of KOA include injuries and inflammation of the knee joint caused by various factors. These long-term injuries could induce articular cartilage degeneration [3]. KOA mainly occurs in middle-aged or elderly individuals, and its progression may result in severe complications such as pain and loss of mobility of the knee joint $[4,5]$.

Extensive studies have investigated the epidemiology and treatment of KOA; nevertheless, there is no consensus on the ideal therapy. As KOA is multifactorial, it is influenced by local, systemic, and external factors and its response to treatment or progression show wide range of variation between patients [6]. It is also a progressive condition, and regression of the condition and restoration is unlikely. Thus, most current 
treatment approaches tend to focus on management of symptoms. Several treatments with varying clinical efficacies have been applied for KOA. Different guidelines and recommendations have been put forth by different professional bodies, such as the Osteoarthritis Research Society International (OARSI), American College of Rheumatology (ACR), and the American Academy of Orthopedic Surgeons (AAOS).

Recommendations for treatment of KOA include transcutaneous electrical nerve stimulation (TENS), exercise in water and on land, weight control, oral nonsteroidal anti-inflammatory drugs (NSAIDs) [7]. In recent years, deep thermotherapy (external high-frequency thermotherapy) has been used to treat malignant tumors, and exhibited superior clinical efficacies for aseptic inflammation [8]. High-frequency ultrasound has emerged as a novel tool to monitor the effectiveness of KOA treatment due to its several advantages such as convenience, speed, non-irradiative property, and ability to be used for the dynamic monitoring of clinical efficacy [9]. Dynamic traction can adjust the line and angle at which the patient's knee is stressed in patients with KOA.

The present study attempted to investigate the effectiveness of deep thermotherapy combined with dynamic traction in patients with KOA after diagnosis with high-frequency ultrasound.

\section{Patients And Methods \\ Patients}

The study prospectively enrolled 60 consecutive patients with KOA who were treated at the Rehabilitation Department of Cangzhou Hospital of Integrated TCM-WM, Hebei between May 2018 and December 2019.

The inclusion criteria were: 1) clinical manifestations including pain, stiffness, and swelling in the knee joint for less than 5 months; 2) no history of joint surgery; and 3) with unilateral grade III/IV KOA on radiological assessment using Kellgren-Lawrence X-ray grading standard. The Kellgren-Lawrence X-ray grading standard is as follows [10]: Grade 0: normal; Grade I: suspicious narrowing of the joint space and suspicious osteophytes; Grade II: suspicious narrowing of the joint space and obvious osteophytes; grade III: significant narrowing of the joint space, moderate osteophytes, and some bone sclerosis or wear; Grade IV: significant narrowing of the joint space, numerous osteophytes, severe bone sclerosis, and obvious wear and deformity.

The exclusion criteria were: 1) severe cardiocerebrovascular diseases or organ failure who could not tolerate stimulation; 2) psychiatric diseases; 3 ) neuromuscular conditions (such as stroke, brain trauma, spinal cord injury, spinal diseases, cerebral palsy, and Parkinson's disease) leading to abnormal gait; 4) tumor, tuberculosis, infection, or rheumatoid diseases; 5 ) disease of the vertebra, hip, ankle, or feet that could influence the gait measurement; or 6) joint pain preventing walking autonomously and requiring assistance.

This study was approved by the Ethics Committee of Cangzhou Hospital of Integrated TCM-WM, Hebei, and informed consent was obtained from all patients.

\section{Diagnostic criteria for KOA}


KOA was diagnosed according to the "Guidelines for the Diagnosis and Treatment of Osteoarthritis (2007 edition)" that were revised and issued by the Chinese Orthopaedic Association [5]: 1) repeated knee joint pain within the past month; 2) X-ray (standing position or weight-bearing position) exhibiting reduced joint space, osteosclerosis, or cystic degeneration of subchondral bone, and osteophyte formation at joint margin; 3) clear and sticky synovial fluid (in at least two measurements) with $\mathrm{WBC}<2000 / \mathrm{mL}$; 4) middleaged or elderly patients ( $\geq 40$ years old); 5 ) morning stiffness $\leq 3 \mathrm{~min}$; and 6 ) bony crepitus during activities. The patients were diagnosed with KOA if they met the items of $1+2,1+3+5+6$, or $1+4+5+6$.

\section{Rehabilitation treatments}

Regular skin traction was performed for the rehabilitation treatment. The fixation point was set at the ankles, using traction weight as the weight-bearing material. The traction weight was placed on the expansion piece to allow the adjustment of the interval time. The traction frame was fixed to the end of the deep thermotherapy bed, and the traction angle $\left(60^{\circ}-80^{\circ}\right)$ was selected according to the severity of knee joint movement limitation, joint space, and biomechanical direction. The weight of traction was $4-6 \mathrm{~kg}$ according to the muscle strength and tolerability of the patients. An ex-vivo high-frequency thermotherapy machine (HG-2000Il, HoKai, Zhuhai, China) was used for ex-vivo heating, with the ring electrode placed at the knee joint. The frequency was set at $13.56 \mathrm{MHz}$ to generate high-frequency oscillation (HFO) by high frequency electromagnetic field, and thus apply vertical radiation to the joint to allow heating. For the deep thermotherapy under dynamic traction, the patients were placed in sitting position, and the knee joint expansion piece was set as follows: traction $10 \mathrm{~s}$ and resting $10 \mathrm{~s}$. Each thermotherapy lasted $30 \mathrm{~min}$, and one treatment was performed per day. One cycle constituted 10 days of therapy, and the patients were treated for a total of 4 cycles.

\section{Procedures for high-frequency ultrasound examination}

The MYLAB60 color Doppler ultrasound diagnostic apparatus and the equipped probes (Esaote, Italy) were used for examination. The frequency was set at $5-13 \mathrm{~Hz}$. The patients were placed in the supine position for examination, the knee joint was bended at $30^{\circ}-90^{\circ}$ to the bed board, and then the cross-sections of the knee joint were scanned clockwise. The patients were then placed in the prone position, and the entire popliteal fossa was scanned by the probe. The joint effusion was evaluated according to the Ribbens diagnostic criteria [11], with joint effusion considered if the depth of anechoic area in the suprapatellar bursa was $\geq 1 \mathrm{~mm}$ and fluid sonolucent area appeared at the tendon of quadriceps femoris muscle and anterior of the lower femoral bone. The blood flow for the synovium was classified into four grades according to the Alder criteria [12] (Supplementary Table 1).

\section{Outcomes}

High-frequency ultrasound was used to assess the pretreatment and 20- and 40-day post-treatment knee joint effusion, thickness of the synovium, and blood flow signal in the synovium. The visual analog scale (VAS) was used to evaluate the pain intensity in the knee joint [13]. The Lysholm scoring system [14], which consisted of questions regarding eight items including limping, supporting, leg scissors, stability, pain, swelling, climbing, and squatting, was used to evaluate knee joint functions. The total possible score was 100 points, and a higher score was indicative of better knee joint function. 


\section{Statistical analysis}

Statistical analysis was performed using SPSS 21.0 software. Quantitative data were described as means \pm standard divisions and compared using one-way repeated measures analysis of variance followed by posthoc pair-wise comparison (Bonferroni). Qualitative data were described as percentages (\%) and compared using non-parametric rank-sum test. $\mathrm{P}<0.05$ was considered statistically significant. The sample size was calculated using the formula $N=\left[\left(Z_{\alpha / 2}+Z_{\beta}\right) \sigma / \delta\right]^{2}$, where $\sigma=9.45, \delta=3.7, a=0.05, \beta=0.10$, one side, and $N$ $=57 . \sigma$ was the estimated standard deviation of the 40-day post-treatment Lysholm score minus the preoperative Lysholm score, and $\delta$ was the permissible error. Considering a lost-to-follow-up rate of about $15 \%$, we planned to enroll 67 patients.

\section{Results}

\section{General characteristics of the patients}

Initially, 67 patients were recruited according to the inclusion criteria. Of these, 2 were excluded due to history of stroke, and 5 were lost to follow up due to treatment discontinuation and early discharge. Thus, 60 patients were included in the analysis. There were 37 (61.7\%) men and 23 (38.3\%) women. The mean age of the patients was 58 (range: 50-69) years. The general characteristics of the 60 patients are presented in Table 1.

Table 1

General characteristics of the patients

\begin{tabular}{|ll|}
\hline Characteristics & $\mathbf{N}=60$ \\
\hline Age (years) & $58(50-69)$ \\
\hline Sex (male) & $37(61.6 \%)$ \\
\hline Affected side (right) & $27(45.0 \%)$ \\
\hline KOA course (months) & $29(12-47)$ \\
\hline
\end{tabular}

\section{Comparison of the pre- and post-treatment VAS and Lysholm scores}

The 20-day post-treatment Lysholm score increased by 16.05 points [ $95 \%$ confidence interval (Cl): $14.92-$ 17.18 points] than pre-treatment score $(P<0.001)$, and the 40-day post-treatment score increased by 18.76 points (95\% Cl: $17.64-19.89)$ at than the 20 -day post-treatment score $(\mathrm{P}<0.001)$. The 20 -day posttreatment VAS score decreased by 2.75 points ( $95 \% \mathrm{Cl}$ : $2.53-2.97$ points) relative to the pre-treatment score $(\mathrm{P}<0.001)$, and the 40 -day post-treatment score decreased by 2.32 points $(95 \% \mathrm{Cl}: 2.10-2.54)$ relative to the 20-day post-treatment score $(P<0.001)($ Table 2$)$. 
Table 2

Knee function and soft tissue changes before and after the treatment $(n=60)$

\begin{tabular}{|c|c|c|c|c|c|c|c|c|}
\hline & $\begin{array}{l}\text { Before } \\
\text { treatment }\end{array}$ & $\begin{array}{l}20 \mathrm{~d} \text { after } \\
\text { treatment }\end{array}$ & $\begin{array}{l}\text { 40d after } \\
\text { treatment }\end{array}$ & $F$ & $\begin{array}{l}\text { Partial } \\
\text { १2 }\end{array}$ & $P$ & $\begin{array}{l}\Delta_{1} \\
(95 \% \mathrm{Cl})\end{array}$ & $\begin{array}{l}\Delta_{2} \\
(95 \% \mathrm{Cl})\end{array}$ \\
\hline $\begin{array}{l}\text { Lysholm } \\
\text { score }\end{array}$ & $\begin{array}{l}47.17 \pm \\
5.07\end{array}$ & $\begin{array}{l}63.22 \pm \\
3.44^{\mathrm{a}}\end{array}$ & $\begin{array}{l}81.98 \pm \\
3.30^{\mathrm{ab}}\end{array}$ & 1391.082 & 0.959 & $\begin{array}{l}< \\
0.001\end{array}$ & $\begin{array}{l}16.05 \\
(14.92- \\
17.18)\end{array}$ & $\begin{array}{l}18.76 \\
(17.64- \\
19.89)\end{array}$ \\
\hline VAS score & $\begin{array}{l}5.67 \pm \\
0.91\end{array}$ & $\begin{array}{l}2.92 \pm \\
0.81^{a}\end{array}$ & $\begin{array}{l}0.60 \pm \\
0.72^{\mathrm{ab}}\end{array}$ & 936.145 & 0.912 & $<0.001$ & $\begin{array}{l}2.75 \\
(2.53- \\
2.97)\end{array}$ & $\begin{array}{l}2.32 \\
(2.10- \\
2.54)\end{array}$ \\
\hline $\begin{array}{l}\text { Joint } \\
\text { effusion } \\
\text { (mm) }\end{array}$ & $\begin{array}{l}5.41 \pm \\
0.46\end{array}$ & $\begin{array}{l}4.34 \pm \\
0.34^{\mathrm{a}}\end{array}$ & $\begin{array}{l}2.32 \pm \\
0.32^{\mathrm{ab}}\end{array}$ & 2010.413 & 0.971 & $\begin{array}{l}< \\
0.001\end{array}$ & $\begin{array}{l}1.07 \\
(0.97- \\
1.17)\end{array}$ & $\begin{array}{l}2.02 \\
(1.95- \\
2.10)\end{array}$ \\
\hline $\begin{array}{l}\text { Thickness } \\
\text { of } \\
\text { synovium } \\
(\mathrm{mm})\end{array}$ & $\begin{array}{l}3.44 \pm \\
0.31\end{array}$ & $\begin{array}{l}2.79 \pm \\
0.15^{a}\end{array}$ & $\begin{array}{l}2.32 \pm \\
0.32^{\mathrm{ab}}\end{array}$ & 283.738 & 0.828 & $\begin{array}{l}<.001 \\
0.001\end{array}$ & $\begin{array}{l}0.65 \\
(0.58- \\
0.72)\end{array}$ & $\begin{array}{l}0.47 \\
(0.37- \\
0.57)\end{array}$ \\
\hline
\end{tabular}

$\Delta_{1}$ : differences between $20 \mathrm{~d}$ after treatment and before treatment; $\Delta_{2}$ : differences between $40 \mathrm{~d}$ after treatment and $20 \mathrm{~d}$ after treatment and before treatment.

a: $\mathrm{P}<0.05$ compared with Before treatment; $\mathrm{b}: \mathrm{P}<0.05$ compared with 20 days after treatment.

\section{Comparison of the pre- and post-treatment joint effusion and synovial thickness}

The 20-day post-treatment joint effusion decreased by $1.07 \mathrm{~mm}(95 \% \mathrm{Cl}: 0.97-1.17)(P<0.001)$ relative to the pre-treatment value, and the 40-day post-treatment value decreased by $2.02 \mathrm{~mm}$ (95\% Cl: 1.95-2.10) (P $<0.001$ ) relative to the 20 -day post-treatment value.

The 20-day post-treatment synovial thickness decreased by $0.65 \mathrm{~mm}(95 \% \mathrm{Cl}: 0.58-0.72)(\mathrm{P}<0.001)$ relative to the pre-treatment, and the 40 -day post-treatment value decreased by $0.47 \mathrm{~mm} \mathrm{(95 \%} \mathrm{Cl:} 0.37-0.57)$ $(P<0.001)$ relative to the 20 -day post-treatment value (Table 2 ).

\section{Comparison of the grade of blood flow signal in synovium before and after the treatment}

The pre-treatment and 20- and 40-day post-treatment grades of synovial blood flow signal exhibited a significant difference $(P<0.001)$ (Table 3). Additionally, the grade of blood flow signal in the synovium at 20-day post-treatment significantly differed from the pre-treatment grade $(P=0.030)$. However, the posttreatment grade of blood flow signal in the synovium did not significantly differ between 20 and 40 days $(P$ $=0.055)$. 
Table 3

Blood flow signal in synovium before and after the treatment $(n=60)$

\begin{tabular}{|c|c|c|c|c|c|}
\hline Grade & Before treatment & $20 \mathrm{~d}$ after treatment & $40 \mathrm{~d}$ after treatment & $\mathbf{F}$ & $\mathbf{P}$ \\
\hline 0 & $28(46.7 \%)$ & $39(65.0 \%)$ & $48(80.0 \%)$ & \multirow[t]{4}{*}{16.162} & \multirow[t]{4}{*}{$<0.001$} \\
\hline 1 & $17(28.3 \%)$ & $13(21.7 \%)$ & $9(15.0 \%)$ & & \\
\hline 2 & $12(20.0 \%)$ & $8(13.3 \%)$ & $3(5 \%)$ & & \\
\hline 3 & $3(5.0 \%)$ & $0(0 \%)$ & $0(0 \%)$ & & \\
\hline
\end{tabular}

\section{Discussion}

A total of 60 patients with KOA were treated with deep thermotherapy combined with dynamic traction in this study. The results revealed significant increases in the post-treatment Lysholm score and significant decreases in the post-treatment VAS scores (20-and 40-day post-treatment scores; $\mathrm{P}<0.001$ in both cases) relative to the pre-treatment values, indicating that the thermotherapy combined with dynamic traction led to significant improvements in knee joint function and significant reduction in pain, respectively. Similarly, results of the high-frequency ultrasound revealed a significant difference in the grade of synovial blood flow signal between the pre- and post-treatment grades, a significant decrease in synovial thickness at 20 and 40 days post-treatment relative to the pretreatment value $(P<0.001)$, and significant decrease in joint effusion at 20 and 40 days post-treatment relative to the pretreatment score $(P<0.001)$.

Currrently, evidence for the use of heat in treating KOA is limited. However, cold application for KOA has been shown to decrease pain, increase joint range, improve the knee's functional capacity, and increase quality of life $[15,16]$. In a study on 100 patients with bilateral KOA treated with short-wave diathermy, the patients exhibited significant decreases in the VAS and Lequesne index scores and better muscle strength compared to patients in the control group [17]. In one study, 110 patients with bilateral primary KOA were received physical treatments with or without heat, diathermy, and ultrasound, and found better improvement in the heat-treated group [18]. However, that study also found no difference in pain and functional parameters in patients treated with short-wave diathermy but instead found improvements in the group treated with hot packs. These results suggest that the approach for heat treatment (i.e. hot pack, short-wave diathermy, or high-frequency thermotherapy) may also influence how the heat is generated and transmitted to the knee joints, consequently affecting the results.

X-ray, computed tomography (CT) scanning, and magnetic resonance imaging (MRI) have been currently used to evaluate effectiveness of treatment of KOA [19]. However, these methods have several limitations. For example, CT scans do not provide much information about cartilage status unless advanced OA has already set in [20]. High-frequency ultrasound examination is more useful to visualize non-bony structures than X-ray [21] because it can be used to observe changes in lesions and blood supply from various angles, and can thus evaluate the pathological changes of KOA more comprehensively [22]. Additionally, it is more rapid and cost-effective than MRI, and can be performed repeatedly. However, it is highly techniquesensitive, and the operator's skill could influence the examinations and evaluations substantially. Thus, 
improving operator skills and standardizing the evaluation procedures could effectively improve the accuracy of evaluation. Azma et al. [23] and Qvistgaard et al. [24] exhibited that high-frequency ultrasound could accurately detect lesions in articular cavities and display the conditions of intercondylar cartilage, synovium, and surrounding soft tissues. Wan et al. [25] and Huang et al. [26] demonstrated that highfrequency ultrasound could directly display the severity of knee joint cartilage and the lesion characteristics of the synovium and bursa mucosa of the knee joint during rehabilitation treatment of arthritis of the knee joint, assisting in the early detection of cartilage diseases. They also demonstrated that high-frequency ultrasound could be used to measure the depth of joint effusion and synovial thickness and evaluate KOA rehabilitation. Therefore, we used high-frequency ultrasound in the present study to determine joint effusion, synovial thickness, and synovial blood flow grade to evaluate the effectiveness of the treatment.

The present study exhibited that deep thermotherapy combined with dynamic traction could significantly reduce the pain intensity of KOA patients. Cai et al. [27] reported that the degree of both pain and swelling in patients with KOA improved significantly after traction combined with Pu garlic moxibustion, which were in agreement with the findings of this study. Ma et al. [28] used high-frequency ultrasound to observe the preand post-treatment changes in KOA treated with needle-knife therapy, and observed that the VAS score decreased and that the joint effusion, synovial thickness, and synovial blood flow improved significantly after the therapy, which were concurrent with our findings. The present study exhibited that joint effusion and thickness of synovium and synovial blood flow signal decreased significantly. These findings were mirrored by a study by Guo et al. [29], suggesting that high-frequency ultrasound could provide objective evidence to evaluate changes in disease condition and to determine treatment efficacy.

The present study used intermittent dynamic constant-force skin traction combined with deep thermotherapy, which could assist the exertion of the effects of deep thermotherapy. Alternating between traction and relaxation could allow the high-frequency electromagnetic field to influence tissue fluids[30], thus increasing the flow exchange rate, blood circulation, the phagocytic ability of white blood cells, the rate of tissue repair, reducing the tension in muscles and connective tissues surrounding the knee joint [31]. The traction could immobilize and rest the affected limb, and also utilize the creep properties of viscoelastic bodies (such as ligaments and muscles), releasing the spasm of muscles surrounding the knee joint, increasing joint space, reducing intra-joint pressure, increasing the range of joint motion, adjusting the line of biomechanical force of the joint [32]. Thus, pain was reduced, and knee joint functions and physiological conditions of the soft tissues were improved.

This study has several limitations. First, this was a single-center study with a small sample size. Second, this is a one-arm study with no control group. Third, the follow-up time of this study was relatively short. Future multicenter randomized control trials with larger sample sizes and longer follow-up durations can help verify the findings of the present study.

Therefore, the effectiveness of deep thermotherapy combined with dynamic traction is evident in treating KOA. High-frequency ultrasound has evident advantages in evaluating the treatment outcomes, and joint effusion, thickness of synovium, and blood flow signal in synovium could be indicators for accurate evaluation. 


\section{List Of Abbreviations}

knee osteoarthritis (KOA)

visual analog scale (VAS)

osteoarthritis (OA)

American College of Rheumatology (ACR)

American Academy of Orthopedic Surgeons (AAOS)

transcutaneous electrical nerve stimulation (TENS)

nonsteroidal anti-inflammatory drugs (NSAIDs)

computed tomography (CT)

magnetic resonance imaging (MRI)

\section{Declarations}

\section{Ethics approval and consent to participate}

This study was approved by the Ethics Committee of Cangzhou Hospital of Integrated TCM-WM, Hebei, and informed consent was obtained from all patients. All methods were carried out in accordance with relevant guidelines and regulations, KOA was diagnosed according to the "Guidelines for the Diagnosis and Treatment of Osteoarthritis (2007 edition)" that were revised and issued by the Chinese Orthopaedic Association.

\section{Consent for publication}

Not applicable.

\section{Availability of data and materials}

The datasets used and/or analysed during the current study available from the corresponding author on reasonable request.

\section{Competing interests}

The authors declare no conflict of interest. 


\section{Funding}

None.

\section{Authors' contributions}

Lichun Wang and Hongli Zhang carried out the studies, participated in collecting data, and drafted the manuscript. Liu Lv and Hua Chen and Jianyong Zhao performed the statistical analysis and participated in its design. Kaixuan Yao and Xiaochen Feng and Fei Li helped to draft the manuscript. All authors read and approved the final manuscript.

\section{Acknowledgements}

None.

\section{References}

1. Vina ER, Kwoh CK. Epidemiology of osteoarthritis: literature update. Curr Opin Rheumatol. 2018;30:1607. doi: $10.1097 /$ BOR.0000000000000479

2. Zhang Y, Jordan JM. Epidemiology of osteoarthritis. Clin Geriatr Med. 2010;26:355-69. doi: 10.1016/j.cger.2010.03.001

3. Yan A, Zhang K, Qin WK, Guo JZ, Li LH, Chen M, et al. [Clinical effects of rehabilitation exercise in the treatment of knee osteoarthritis based on the theory of "treating muscle for bone"]. Zhongguo Gu Shang. 2017;30:731-4. doi: 10.3969/j.issn.1003-0034.2017.08.010

4. Filippiadis D, Charalampopoulos G, Mazioti A, Alexopoulou E, Vrachliotis T, Brountzos E, et al. Interventional radiology techniques for pain reduction and mobility improvement in patients with knee osteoarthritis. Diagn Interv Imaging. 2019;100:391-400. doi: 10.1016/j.diii.2019.02.011

5. Sadatsuki R, Ishijima M, Kaneko H, Liu L, Futami I, Hada S, et al. Bone marrow lesion is associated with disability for activities of daily living in patients with early stage knee osteoarthritis. J Bone Miner Metab. 2019;37:529-36. doi: 10.1007/s00774-018-0950-z

6. Mora JC, Przkora R, Cruz-Almeida Y. Knee osteoarthritis: pathophysiology and current treatment modalities. J Pain Res. 2018;11:2189-96. doi: 10.2147/JPR.S154002

7. Kan HS, Chan PK, Chiu KY, Yan CH, Yeung SS, Ng YL, et al. Non-surgical treatment of knee osteoarthritis. Hong Kong Med J. 2019;25:127-33. doi: 10.12809/hkmj187600

8. Zhang H, Lv L, Wang L. Controlled Study of Traction Combined with Deep Hyperthermia for Knee Osteoarthritis Based on Plantar Pressure Measurement. Feng Shi Bing Yu Guan Jie Yan. 2019;10:14-8.

9. Huang YP, Zhong J, Chen J, Yan CH, Zheng YP, Wen CY. High-Frequency Ultrasound Imaging of Tidemark In Vitro in Advanced Knee Osteoarthritis. Ultrasound Med Biol. 2018;44:94-101. doi: 10.1016/j.ultrasmedbio.2017.08.1884 
10. Kellgren JH, Lawrence JS. Radiological assessment of osteo-arthrosis. Ann Rheum Dis. 1957;16:494502. doi: 10.1136/ard.16.4.494

11. Conaghan PG, McGonagle D, Wakefield R, Emery P. New approaches to imaging of early rheumatoid arthritis. Clin Exp Rheumatol. 1999;17:S37-42.

12. Paolillo FR, Paolillo AR, Joao JP, Frasca D, Duchene M, Joao HA, et al. Ultrasound plus low-level laser therapy for knee osteoarthritis rehabilitation: a randomized, placebo-controlled trial. Rheumatol Int. 2018;38:785-93. doi: 10.1007/s00296-018-4000-x

13. McCormack HM, Horne DJ, Sheather S. Clinical applications of visual analogue scales: a critical review. Psychol Med. 1988;18:1007-19. doi: 10.1017/s0033291700009934

14. Kun H, Lin M, Liu J. Progress on Tai Chi Therapy for Patients with Senile Knee Osteoarthritis. Rehabilitation Medicine. 2017;27:59.

15. Román-Belmonte J, De la Corte-Rodríguez H, Rodríguez-Merchán E: Physical Medicine and Rehabilitation in Knee Osteoarthritis. In: Comprehensive Treatment of Knee Osteoarthritis. edn.: Springer, Cham; 2020: 11-24.

16. Aciksoz S, Akyuz A, Tunay S. The effect of self-administered superficial local hot and cold application methods on pain, functional status and quality of life in primary knee osteoarthritis patients. J Clin Nurs. 2017;26:5179-90. doi: 10.1111/jocn.14070

17. Cetin N, Aytar A, Atalay A, Akman MN. Comparing hot pack, short-wave diathermy, ultrasound, and TENS on isokinetic strength, pain, and functional status of women with osteoarthritic knees: a singleblind, randomized, controlled trial. Am J Phys Med Rehabil. 2008;87:443-51. doi:

10.1097/PHM.0b013e318174e467

18. TERZI R, ALTIN F. Evaluation of Short-Wave Diathermy and Ultrasound Treatments as Combined Physical Treatments for Knee Osteoarthritis Journal of Physical Medicine \& Rehabilitation Sciences/Fiziksel Tup ve Rehabilitasyon Bilimleri Dergisi. 2017;20.

19. Wang MY, Wang XB, Sun XH, Liu FL, Huang SC. Diagnostic value of high-frequency ultrasound and magnetic resonance imaging in early rheumatoid arthritis. Exp Ther Med. 2016;12:3035-40. doi: 10.3892/etm.2016.3695

20. Liu M. Evaluating the effectiveness of warm needling method in treating knee osteoarthritis. Lin Chuang Yi Yao Wen Xian Dian Zi Za Zhi. 2017;4:12331-2.

21. Wang J. Comparison of the values of high-frequency ultrasound and X-ray for the diagnosis of knee arthritis He Bei Yi Yao. 2017;22:3452-4.

22. Zhong G, Xie C. Comparison of the performance of high-frequency ultrasound and X-ray for knee osteoarthritis. Jiang Xi Yi Yao. 2020;01:88-90.

23. Azma K, RezaSoltani Z, Rezaeimoghaddam F, Dadarkhah A, Mohsenolhosseini S. Efficacy of telerehabilitation compared with office-based physical therapy in patients with knee osteoarthritis: $A$ randomized clinical trial. J Telemed Telecare. 2018;24:560-5. doi: 10.1177/1357633X17723368

24. Qvistgaard E, Kristoffersen H, Terslev L, Danneskiold-Samsoe B, Torp-Pedersen S, Bliddal H. Guidance by ultrasound of intra-articular injections in the knee and hip joints. Osteoarthritis Cartilage. 2001;9:512- 
7. doi: $10.1053 /$ joca.2001.0433

25. Wan R, Zhong N, Feng N. Value of using high-frequency ultrasound to evaluate the cartilage changes and para-knee tissues in patients with knee osteoarthritis. Zhong Guo Lao Nian Xue Za Zhi. 2016;21:5409-10.

26. Huang S, Lin Y, Zhong F. The value of high frequency ultrasound in evaluation of knee osteoarthritis. Xian Dai Yi Yong Ying Xiang Xue. 2018;07:2218-20.

27. Cai D, Xiong H, Cai W, Ding P. Evaluating the effectiveness of knee joint traction combined with $\mathrm{Pu}$ Garlic Moxibustion for knee osteoarthritis. Lin Chuang Yi Yao Wen Xian Dian Zi Za Zhi. 2015;13:2505-7.

28. Ma Y, Ding K, Wang S. Value of high-frequency ultrasound in evaluating the effects of needle-knife for the treatment of knee osteoarthritis. Xian Dai Zhong Xi Yi Jie He Za Zhi. 2012;10:1074-5.

29. Guo H, Fan J, Xue Y. Application of high frequency ultrasound in knee joint lesions of elderly patients with rheumatoid arthritis Zhong Guo Zong He Lin Chuang. 2017;33:602-5.

30. Zhang Q, Shen M, Yang J. The depth portion thermotherapy unites Terry to the proper treatment chronic prostatitis's curative effect observation. Hu Li Shi Jian Yu Yan Jiu. 2008;5:48-9.

31. Zhou Y, Zhan A, Zeng H. The Preliminary Study of Ultrasound Scoring in Grading Diagnosis of Knee Joint Osteoarthritis. Zhong Guo Chao Sheng Yi Xue Za Zhi. 2018;6.

32. Miao J, Shui G, Wang A. TCM Tazi combined with infrared radiation for the treatment of knee osteoarthritis: clinical observation in 60 patients Zhong Yi Yao Dao Bao. 2014;20:123-4.

\section{Supplementary Files}

This is a list of supplementary files associated with this preprint. Click to download.

- SupplementaryTable1.pdf 